\title{
Analysis of Energy Management of Garnesa Electric Car Based Numerical Simulation Modeling
}

\author{
Bidya Nur Habib ${ }^{1}$, Agung Prijo Budijono ${ }^{2}$ \\ State University of Surabaya \\ bidyaft@gmail.com
}

\begin{abstract}
Designing an electric car to compete with ESCC should be guided by Vehicle Construction and Stability. One of the areas to consider when designing at the research and development stage is the Mechanical Mechanical System (Rotational Mechanical System). These systems include, wheels, transmissions (gear connections), electric motor rotors and shafts. The purpose of this study was to determine the effect of vehicle energy management on driver driving strategies during the ESCC competition. This is based on Wheel Mechanical System modeling, Dynamic System Analysis and Free Body Diagram. The method used is based on Numerical Simulation. The data parameters used are based on independent variables and control variables. The independent variable of this study is the angular velocity $(\mathrm{V} \omega)$, linear velocity (v) of the vehicle, friction coefficient value (B), shaft stiffness (K), wheel diameter, gear diameter, wheel mass and moment inertia of the wheel. Control variables is technical regulation of ESCC Urban Concept. This Numerical Simulation Test is to determine the required electrical power, travel time and distance of the vehicle. The results showed that the energy needed by GARNESA electric car with an average speed selection of $9.42 \mathrm{~m} / \mathrm{s}$ based on a maximum speed of $10.15 \mathrm{~m} / \mathrm{s}$ and a minimum speed of $8.70 \mathrm{~m} / \mathrm{s}$ requires the amount of power 248.15 Watt. Travel time is 180 seconds in one lap. The distance obtained is $1357 \mathrm{~m}$. Driving strategy based on average speed of 9.42 $\mathrm{m} / \mathrm{s}$ consumes less power and the distance obtained will be more far.
\end{abstract}

Keywords: Energy Management, KMHE, Numerical Simulation, Free Body Diagram (FBD).

\section{INTRODUCTION}

Electric car becomes the main segment of the market today and in the future because it has significant advantages. It uses an electric motor for propulsion, and batteries as electrical storage device. The electric motor has many advantages such as fast torque respones in milliseconds, easiness in obtaining accurate torque feedback, capable of generating both traction and braking forces and small sizes but powerful output [6-7].Various advantages possessed by this vehicle, among others, it has greater efficiency than conventional ones. The overall efficiency of it about 48 percent, significantly better compared to conventional ones which achieve efficiency of around $25 \%$. Electric car engines are so good that they do not cause noise pollution problems. The cost of refilling it also very affordable. The average electric car requires a refill fee of 2 cents per mile compared to 12 cents per mile on conventional ones. Its lifetime is estimated to be around 90 years, if it is driven fifty miles (around $80 \mathrm{~km}$ ) per day. It also have lower maintenance costs compared to conventional ones because it only have about 5 parts in their motors, compared to traditional ones that have hundreds of components in internal combustion engines. In our country, electric cars have also been developed. One of the efforts made by the Kemenristek DIKTI is to hold an Energy Saving Car Contest (ESCC) each year involving all Universities in Indonesia. Garuda Unesa (GARNESA) is an Urban Concept electric car developed by UNESA to participate in the ESCC. The ability to design and build economical, safe and environmentally friendly vehicles is a mandatory requirement that must be owned by the Team concerned. Designing an electric car must be guided by Construction and Vehicle Stability. Pamungkas et al [2] showed that The designed model is able to describe the dynamic behavior of handling Smart-EV.2 electric cars with a response error between 5-15\%. The 
maximum value of longitudinal acceleration and pitch rate in sudden braking maneuvers is $4.3 \mathrm{~m} / \mathrm{s}^{2}$ and 9 degrees $/ \mathrm{sec}$, while sudden acceleration is $3 \mathrm{~m} / \mathrm{s}^{2}$ and 4.8 degrees $/ \mathrm{sec}$. The time needed to stop from the speed of $60 \mathrm{~km} / \mathrm{h}$ is 4 seconds with a braking distance of $32.4 \mathrm{~m}$. Muhammad Tadjuddin et al [4] claimed about the driving strategies of electric car. Strategy I consumes power of $105.08 \mathrm{~km} / \mathrm{kwh}$ with travel time of 24.83 minutes, in strategy II it consumes power of $144.17 \mathrm{~km} / \mathrm{kwh}$ with travel time of 28.83 minutes, and in strategy III it consumes power of $119.484 \mathrm{~km} / \mathrm{kwh}$ with a travel time of 23.2 minutes. Daziano [1] conclude that an electric vehicle could be perceived as equivalent to a combustion vehicle if its achieved driving range were about 330 miles. Range anxiety has a strong relationship to the journey efficiency and also to the SOC of the battery. The trend suggest that users begin to modify their driving style as the SOC is reduced to less than 50\% [5]. Rauh et al [3] compared 12 motorists with high battery electric vehicle (BEV) driving experience $(60,500 \mathrm{~km})$ with 12 motorists who had never driven a BEV before. They examined range appraisal and range stress (range anxiety) on different levels (cognitive, emotional and behavioral). This study focused on how to make system model of rotational mechanical system with Simulink. This system includes, wheels, transmission (gear connection), electric motor rotor and shaft. Designing the Vehicle Rotation System must refer to the ESCC Guidelines and vehicle construction. The form of system analysis can be done using the simulation method. Calculation and simulation analysis helps researchers in describing vehicle specifications against electrical energy requirements when driving.

\section{METHODOLOGY}

The method used is based on Numerical Simulation Modeling with Simulink. Simulation is done through Wheel Mechanical System modeling, Dynamic System Analysis and Free Body Diagram (FBD).

2.1 Data collection technique

Data retrieval is a process that is important in achieving the research objectives. The data in this study were obtained by inputting data on the MATLAB Software. The data needed is in accordance with the parameters of the independent variable and the control variable that has been designed.

2.2 Data analysis

The technique used to analyze the data in this study is quantitative descriptive statistics. This data analysis technique is carried out by analyzing the data obtained from each change that occurs through a simulation experiment directly, where the results are in the form of quantitative data. 


\section{RESULT AND DISCUSSION}

Block diagram of simulink
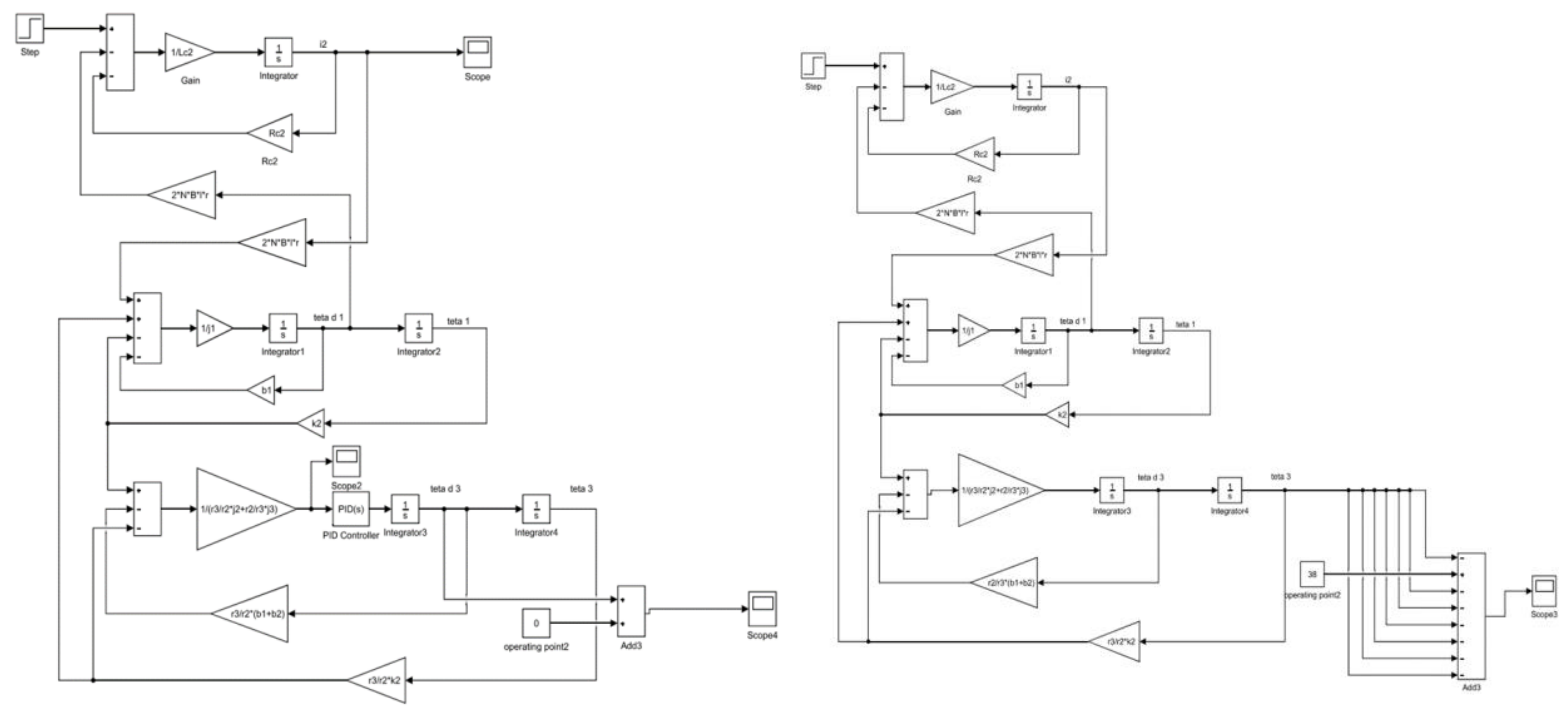

Figure 1. Block diagram of simulink

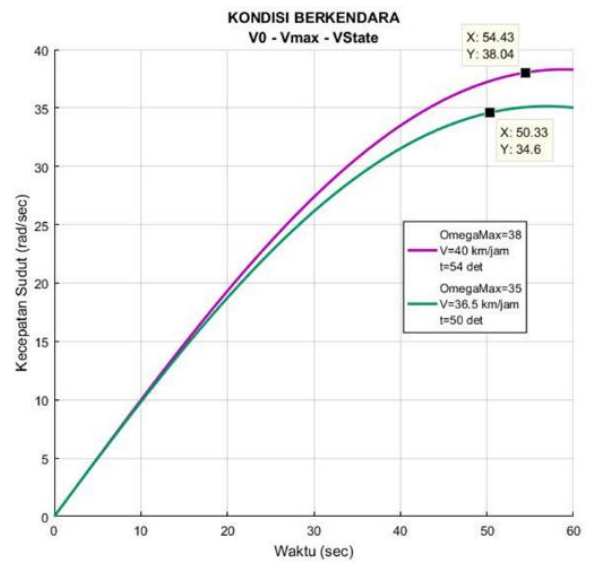

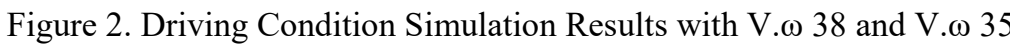

Based on the picture above, it can be seen that if the driver of the GARNESA electric car provides stamping input to reach V. Max $38 \mathrm{rad} / \mathrm{sec}(11.02 \mathrm{~m} / \mathrm{s})$ takes $54.43 \mathrm{sec}$. Instead V. Max $35 \mathrm{rad} / \mathrm{sec}(10.15 \mathrm{~m} / \mathrm{s})$ is taken within $50.33 \mathrm{sec}$. Travel time difference if the driver steps on the gas pedal to a maximum speed of $11.02 \mathrm{~m} / \mathrm{s}$ and $10.15 \mathrm{~m} / \mathrm{s}$ of $4.13 \mathrm{sec}$. 


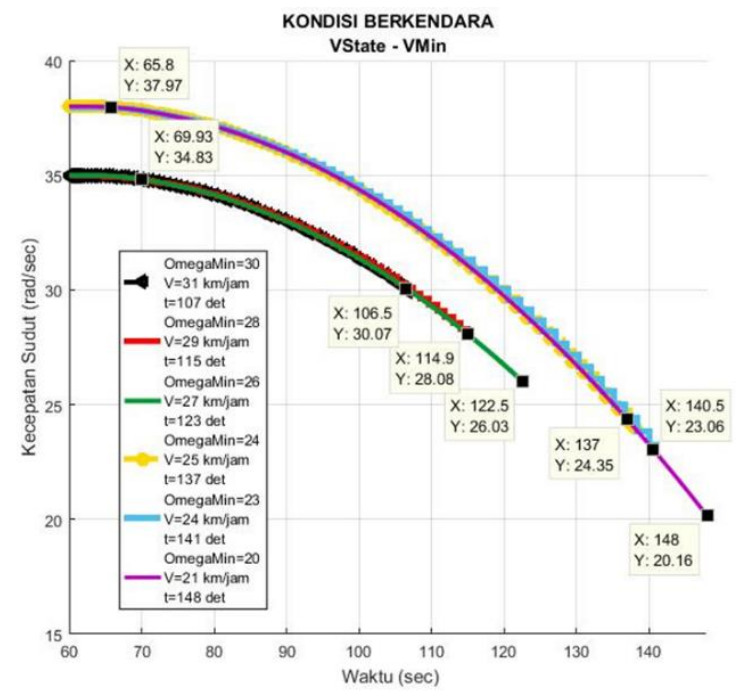

Figure 3. Driving Condition Simulation Results with V.max $\omega 38$ - V.min $\omega 24,23,20$ and V.max $\omega 35-$ V.min $\omega 30,28,26$.

The condition of V.state with Vax $\omega 38 \mathrm{rad} / \mathrm{sec}(11.02 \mathrm{~m} / \mathrm{s})$ is for $11.37 \mathrm{sec}$. Whereas the condition of V.state with V.max $\omega 35 \mathrm{rad} / \mathrm{sec}(10.15 \mathrm{~m} / \mathrm{s})$ is for $19.67 \mathrm{sec}$. The time taken from V.state $\omega 38 \mathrm{rad} / \mathrm{sec}$ to V.min $\omega 24 \mathrm{rad} / \mathrm{sec}(6.96 \mathrm{~m} / \mathrm{s})$ is $71.2 \mathrm{sec}$. The time taken from V.State is $\omega 38 \mathrm{rad} / \mathrm{sec}$ to V.min $\omega 23$ $\mathrm{rad} / \mathrm{sec}(6.67 \mathrm{~m} / \mathrm{s})$ of $75.2 \mathrm{sec}$. The time taken from V.state is $\omega 38 \mathrm{rad} / \mathrm{sec}$ to V.min $\omega 20 \mathrm{rad} / \mathrm{sec}(5.8$ $\mathrm{m} / \mathrm{s})$ of $82.2 \mathrm{sec}$.

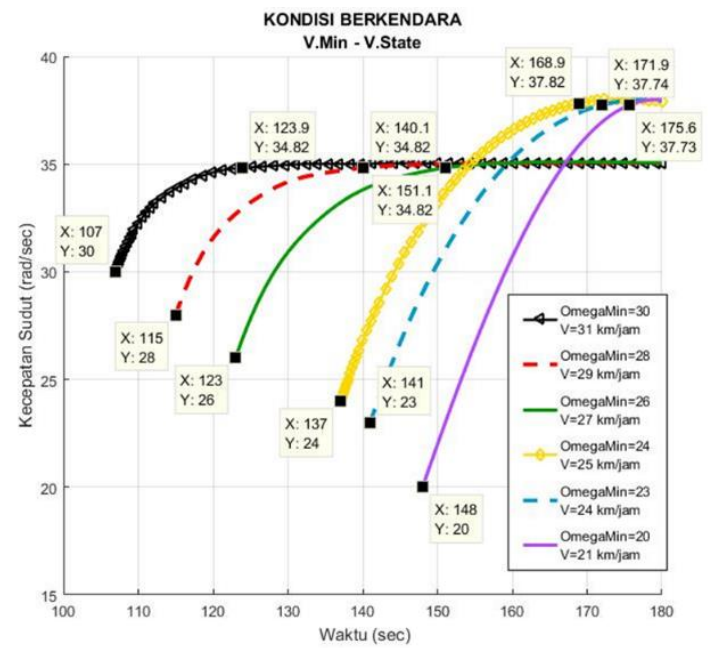

Figure 4. Driving Condition Simulation Result with V.min - V.state

V.min $\omega 20$ towards V.maks $\omega 38$ while driving by $28 \mathrm{sec}$. Whereas V.maks becomes V.state takes 4 sec. The time taken from V.min $\omega 23$ is the menu for V.maks $\omega 38$ of $31 \mathrm{sec}$. Whereas V.Maks becomes V.state takes $8 \mathrm{sec}$. The time taken from V.min $\omega 24$ to V.maks $\omega 38$ is $32 \mathrm{sec}$ and from V.maks to V.state takes 11 sec. V.min $\omega 26$ headed to V.maks $\omega 35$ while driving by $28 \mathrm{sec}$. Whereas.max becomes V.state takes 29 sec. V.min $\omega 28$ headed for V.maks $\omega 35$ while driving by $25 \mathrm{sec}$. Whereas V.maks becomes V.state takes $40 \mathrm{sec}$. V.min $\omega 30$ headed for V.maks $\omega 35$ while driving for $17 \mathrm{sec}$. Whereas V.maks becomes V.state takes $56 \mathrm{sec}$. 
Table 1. Data of energy requirement Vmax $\omega 38$ and Vmin $\omega 24$

\begin{tabular}{|c|c|c|c|c|c|c|c|c|c|c|c|}
\hline \multirow{2}{*}{ Kondisi Berkendara } & \multirow{2}{*}{\multicolumn{2}{|c|}{$\begin{array}{c}\mathrm{V}_{\omega} \\
\text { (radsec) }\end{array}$}} & \multirow{2}{*}{\multicolumn{2}{|c|}{$\begin{array}{c}\text { Vlinear } \\
(\mathrm{m} / \mathrm{s}) \\
\end{array}$}} & \multirow{2}{*}{\multicolumn{2}{|c|}{$\begin{array}{c}\text { Waktu (t) } \\
(\mathrm{s}) \\
\end{array}$}} & \multirow{2}{*}{\begin{tabular}{|c|}
$\mathrm{S}$ \\
$1 \mathrm{x}(\mathrm{m})$ \\
\end{tabular}} & \multirow{2}{*}{\begin{tabular}{|c|} 
Inersia (I) \\
$\mathrm{Kgm}^{\wedge} \mathbf{2}$ \\
\end{tabular}} & \multicolumn{2}{|c|}{\begin{tabular}{|l|l|} 
Kinetik $(€)$ & Daya (P) \\
\end{tabular}} & \multirow{2}{*}{$\frac{\text { Arus (I) }}{\mathrm{A}}$} \\
\hline & & & & & & & & & Joule & Watt & \\
\hline 0 . Vmaks & $0 \cdot 1$ & 38 & 0 . & 11,02 & 0 . & 54,4 & 599,82 & \multirow{2}{*}{18,84} & \multirow{2}{*}{1143,82} & \multirow{2}{*}{96,19} & \multirow{2}{*}{2,00} \\
\hline$\Delta$ & 38 & & 11,02 & & 54,4 & & & & & & \\
\hline Vmaks - Vstate & 38 . & 38 & 11,02 & 11,02 & 54,4 - & 65,8 & \multirow{3}{*}{125,30} & \multirow{3}{*}{18,84} & \multirow{3}{*}{1143,82} & \multirow{3}{*}{96,19} & \multirow{3}{*}{2,00} \\
\hline$\Delta$ & 0 & & 0,00 & & 11,4 & & & & & & \\
\hline Total Vstate & \multicolumn{2}{|l|}{38} & \multicolumn{2}{|c|}{11,02} & \multicolumn{2}{|c|}{11,37} & & & & & \\
\hline Vstate - Vmin & 38. & 24 & 11,02 . & 6,96 & $65,8 \cdot$ & 137 & 289,07 & \multirow{2}{*}{18,84} & \multirow{2}{*}{155,25} & \multirow{2}{*}{13,06} & \multirow{2}{*}{0,27} \\
\hline$\Delta$ & 14 & & 4,06 & & 71,2 & & & & & & \\
\hline \multirow{2}{*}{ Vmin- Vmaks } & 24. & 38 & 6.96 . & 11,02 & 137. & \begin{tabular}{|l|}
169 \\
\end{tabular} & 129,92 & \multirow{2}{*}{18,84} & \multirow{2}{*}{155,25} & \multirow{2}{*}{13,06} & \multirow{2}{*}{0.27} \\
\hline & 14 & & 4,06 & & 32 & & & & & & \\
\hline Vmaks - Vstate & \begin{tabular}{ll|}
$38 \cdot$ \\
\end{tabular} & 38 & 11,02 . & 11,02 & $169 \cdot$ & 180 & \multirow{3}{*}{121,22} & \multirow{3}{*}{18,84} & \multirow{3}{*}{1143,82} & \multirow{3}{*}{96,19} & \multirow{3}{*}{2.00} \\
\hline$\Delta$ & 0 & & 0,00 & & 11 & & & & & & \\
\hline Total Vstate & 38 & & 11 & .02 & 11 & & & & & & \\
\hline
\end{tabular}

Table 2. Data of energy requirement Vmax $\omega 38$ and Vmin $\omega 23$

\begin{tabular}{|c|c|c|c|c|c|c|c|c|c|c|c|}
\hline \multirow{2}{*}{ Kondisi Berkendara } & \multicolumn{2}{|l|}{$v_{\omega}$} & \multicolumn{2}{|c|}{ Vlinear } & \multicolumn{2}{|c|}{ Waktu (t) } & $\mathrm{s}$ & Inersia (I) & Kinetik (t) $\mid$ & Daya (P) & Arus (I) \\
\hline & \multicolumn{2}{|l|}{ (radsec) } & \multicolumn{2}{|c|}{$(\mathrm{m} / \mathrm{s})$} & \multicolumn{2}{|c|}{ (s) } & 1s (m) & $\mathrm{Kgm}^{\prime} 2$ & Joule & Watt & A \\
\hline \multirow[t]{2}{*}{0 -Vmaks } & 0. & 38 & 0 & \begin{tabular}{l|l}
$\cdot$ & 11,02 \\
\end{tabular} & 0. & 54,4 & 599.82 & \multirow{2}{*}{18,84} & \multirow{2}{*}{1143,82} & \multirow{2}{*}{96,19} & \multirow{2}{*}{2,00} \\
\hline & 38 & & 11,02 & & 54,4 & & & & & & \\
\hline \multirow[t]{2}{*}{ Vmaks - Vstate } & 38 - & 38 & 11,02 & \begin{tabular}{l|l}
- & 11,02 \\
\end{tabular} & $54,4 \cdot-$ & 65,8 & \multirow{3}{*}{125,30} & \multirow{3}{*}{18,84} & \multirow{3}{*}{1143,82} & \multirow{3}{*}{96,19} & \multirow{3}{*}{2,00} \\
\hline & 0 & & 0,00 & & 11,4 & & & & & & \\
\hline Total Vstate & \multicolumn{2}{|l|}{38} & \multicolumn{2}{|r|}{11,02} & \multicolumn{2}{|c|}{11,37} & & & & & \\
\hline \multirow[t]{2}{*}{ Vstate - Vmin } & $38 \cdot$ & 23 & 11,02 & 6.67 & $65,8 \cdot$ & 141 & 327,12 & \multirow{2}{*}{18,84} & \multirow{2}{*}{178,23} & \multirow{2}{*}{14,99} & \multirow{2}{*}{0,31} \\
\hline & 15 & & 4,35 & & 75,2 & & & & & & \\
\hline \multirow[t]{2}{*}{ Vmin-Vmaks } & $23 \cdot$ & 38 & 6,67 & $\begin{aligned} &- 11,02 \\
&\end{aligned}$ & 141 - & 172 & 134,85 & \multirow{2}{*}{18,84} & \multirow{2}{*}{178,23} & \multirow{2}{*}{14,99} & \multirow{2}{*}{0,31} \\
\hline & 15 & & 4,35 & & 31 & & & & & & \\
\hline \multirow[t]{2}{*}{ Vmaks - Vstate } & $38 \cdot$ & 38 & 11,02 & $\begin{aligned} &- 11,02 \\
&-\end{aligned}$ & $172 \cdot-$ & 180 & \multirow{3}{*}{88,16} & \multirow{3}{*}{18,84} & \multirow{3}{*}{1143,82} & \multirow{3}{*}{96,19} & \\
\hline & 0 & & 0,00 & & 8 & & & & & & 2,00 \\
\hline Total Vstate & 38 & & & 11,02 & 8 & & & & & & \\
\hline
\end{tabular}

Table 3. Data of energy requirement Vmax $\omega 38$ and Vmin $\omega 20$

\begin{tabular}{|c|c|c|c|c|c|c|c|c|c|c|c|}
\hline \multirow{2}{*}{ Kondisi Berkendara } & \multicolumn{2}{|l|}{$V_{\omega}$} & \multicolumn{2}{|c|}{ Vlinear } & \multicolumn{2}{|c|}{ Waktu (t) } & $\mathrm{s}$ & Inersia (I) & Kinetik $(\epsilon)$ & Daya (P) & Arus (I) \\
\hline & \multicolumn{2}{|l|}{ (rad/sec) } & \multicolumn{2}{|c|}{ (m/s) } & \multicolumn{2}{|r|}{ (s) } & $1 x(\mathrm{~m})$ & $\mathrm{Kgm}^{\prime 2} 2$ & Joule & Watt & A \\
\hline \multirow[t]{2}{*}{0 -Vuaks } & $0 \cdot 2$ & 38 & 0 & \begin{tabular}{r|r}
$\cdot$ & 11,02 \\
\end{tabular} & 0 & \begin{tabular}{|l|l|}
- & 54,4 \\
\end{tabular} & 599,82 & \multirow{2}{*}{18,84} & \multirow{2}{*}{1143,82} & \multirow{2}{*}{96,19} & \multirow{2}{*}{2,00} \\
\hline & \multicolumn{2}{|l|}{38} & \multicolumn{2}{|l|}{11,02} & \multicolumn{2}{|l|}{54,4} & & & & & \\
\hline \multirow[t]{2}{*}{ Vmaks - Vstate } & 38 - & 38 & 11,02 & 11,02 & 54,4 & \begin{tabular}{|l|l|}
- & 65,8 \\
\end{tabular} & \multirow{3}{*}{125,30} & \multirow{3}{*}{18,84} & \multirow{3}{*}{1143,82} & \multirow{3}{*}{96,19} & \multirow{3}{*}{2,00} \\
\hline & \multicolumn{2}{|l|}{0} & 0,00 & & 11,4 & & & & & & \\
\hline Total Vstate & 38 & & \multicolumn{2}{|c|}{11,02} & \multicolumn{2}{|c|}{11,37} & & & & & \\
\hline \multirow[t]{2}{*}{ Vstate - Vmin } & 38 . & 20 & 11,02 & 5,80 & 65,8 & \begin{tabular}{|l|l|}
- & 148 \\
\end{tabular} & 429,08 & \multirow{2}{*}{18,84} & \multirow{2}{*}{256,65} & \multirow{2}{*}{21,58} & \multirow{2}{*}{0,45} \\
\hline & \multicolumn{2}{|l|}{18} & 5,22 & & 82,2 & & & & & & \\
\hline \multirow[t]{2}{*}{ Vmin- Vmaks } & $20 \cdot-1$ & 38 & 5,80 & $\begin{aligned} & \cdot 11,02 \\
&\end{aligned}$ & 148 & \begin{tabular}{|l|l|}
- & 176 \\
\end{tabular} & 146,16 & \multirow{2}{*}{18,84} & \multirow{2}{*}{256,65} & \multirow{2}{*}{21,58} & \multirow{2}{*}{0,45} \\
\hline & \multicolumn{2}{|l|}{18} & 5,22 & & 28 & & & & & & \\
\hline Vmaks - Vstate & $38 \cdot-$ & 38 & 11,02 & 11,02 & 176 & \begin{tabular}{|l|l|}
- & 180 \\
\end{tabular} & & & & & \\
\hline & 0 & & 0,00 & & 4 & & 44,08 & 18,84 & 1143,82 & 96,19 & 2.00 \\
\hline Total Vstate & 38 & & & 1,02 & & 4 & & & & & \\
\hline
\end{tabular}

Table 4. data of energy requierment Vmax $\omega 35$ and Vmin $\omega 30$

\begin{tabular}{|c|c|c|c|c|c|c|c|c|c|c|c|}
\hline \multirow{2}{*}{ Kondisi Berkendara } & \multicolumn{2}{|l|}{$V_{\omega}$} & \multicolumn{2}{|c|}{ Vlinear } & \multicolumn{2}{|c|}{ Waktu (t) } & \multirow{2}{*}{\begin{tabular}{|c|}
$\mathrm{S}$ \\
$1 \mathbf{x}(\mathrm{m})$ \\
\end{tabular}} & \multirow{2}{*}{\begin{tabular}{|c|} 
Inersia (I) \\
Kgm'2 \\
\end{tabular}} & \multicolumn{2}{|c|}{\begin{tabular}{|l|l|} 
Kinetik (f) & Daya (P) \\
\end{tabular}} & \multirow{2}{*}{\begin{tabular}{|c|} 
Arus (I) \\
A
\end{tabular}} \\
\hline & \multicolumn{2}{|c|}{ (radsec) } & \multicolumn{2}{|c|}{ (m/s) } & \multicolumn{2}{|c|}{ (s) } & & & Joule & Watt & \\
\hline \multirow[t]{2}{*}{0 -Vmaks } & 0. & 35 & 0 . & 10,15 & 0. & 50,3 & 0.85 & \multirow{2}{*}{18,84} & \multirow{2}{*}{970,34} & \multirow{2}{*}{81,61} & \multirow{2}{*}{1,70} \\
\hline & 35 & & 10,15 & & 50,3 & & & & & & \\
\hline \multirow[t]{2}{*}{ Vmaks - Vstate } & 35. & 35 & 10,15 . & 10,15 & 50,3 & 70 & \multirow{3}{*}{199,65} & \multirow{3}{*}{18,84} & \multirow{3}{*}{970,34} & \multirow{3}{*}{81,61} & \multirow{3}{*}{1,70} \\
\hline & 0 & & 0,00 & & 19,7 & & & & & & \\
\hline Total Vstate & \multicolumn{2}{|l|}{35} & \multicolumn{2}{|c|}{10.15} & \multicolumn{2}{|c|}{19,67} & & & & & \\
\hline Vstate - Vmin & 35. & 30 & 10,15 - & 8.70 & $\begin{array}{lll}70 . & - \\
\end{array}$ & 107 & 53,65 & \multirow{2}{*}{18,84} & \multirow{2}{*}{19,80} & \multirow{2}{*}{1,67} & \multirow{2}{*}{0,03} \\
\hline & 5 & & 1,45 & & 37 & & & & & & \\
\hline \multirow[t]{2}{*}{ Vmin - Vmaks } & \begin{tabular}{l|l|}
30 & - \\
\end{tabular} & 35 & 8,70 - & 10,15 & 107 - & 124 & 24,65 & \multirow{2}{*}{18,84} & \multirow{2}{*}{19,80} & \multirow{2}{*}{1,67} & \multirow{2}{*}{0,03} \\
\hline & 5 & & 1.45 & & 17 & & & & & & \\
\hline \multirow[t]{2}{*}{ Vinnks - Vstate } & $35 \cdot$ & 35 & 10,15 - & 10,15 & 124. & 180 & \multirow{3}{*}{568,40} & & & & \\
\hline & 0 & & 0.00 & & 56 & & & 18,84 & 970,34 & 81,61 & 1,70 \\
\hline Total Vstate & 35 & & 10. & & 56 & & & & & & \\
\hline
\end{tabular}


Table 5. Data of energy requirement Vmax $\omega 35$ and Vmin $\omega 28$

\begin{tabular}{|c|c|c|c|c|c|c|c|c|c|c|}
\hline \multirow{2}{*}{ Kondisi Berkendara } & \multicolumn{2}{|l|}{$\mathrm{v}_{\omega}$} & \multicolumn{2}{|c|}{ Vlinear } & Waktu (t) & $\mathrm{s}$ & \multirow{2}{*}{\begin{tabular}{|c} 
Inessia (I) \\
Kgm'2 \\
\end{tabular}} & \multicolumn{3}{|c|}{ Kinetik (E) Daya (P) Auvs (I) } \\
\hline & \multicolumn{2}{|c|}{ (nadsec) } & \multicolumn{2}{|r|}{ (ms) } & (s) & $1 x$ (m) & & Joule & Watt & A \\
\hline \multirow[t]{2}{*}{0 -Vmaks } & 0. & 35 & & \begin{tabular}{|l|l|} 
& 10,15 \\
\end{tabular} & \begin{tabular}{l|l|l|}
0 & - & 50,3 \\
\end{tabular} & 510,85 & \multirow{2}{*}{18,84} & \multirow{2}{*}{970,34} & \multirow{2}{*}{81,61} & \multirow{2}{*}{1,70} \\
\hline & 35 & & 10,15 & & 50,3 & & & & & \\
\hline Vmaks - Vstate & 35 . & 35 & 10,15 & 10,15 & 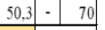 & \multirow{3}{*}{199,65} & \multirow{3}{*}{18,84} & \multirow{3}{*}{970,34} & \multirow{3}{*}{81,61} & \multirow{3}{*}{1,7} \\
\hline$\Delta$ & 0 & & 0,00 & & 19,7 & & & & & \\
\hline Total Vstate & \multicolumn{2}{|l|}{35} & \multicolumn{2}{|r|}{10,15} & 19,67 & & & & & \\
\hline Vstate - Vmin & $35 \cdot-$ & 28 & 10,15 & 8,12 & $70-|115|$ & 91,35 & \multirow{2}{*}{18,84} & \multirow{2}{*}{38,81} & \multirow{2}{*}{3,26} & \multirow{2}{*}{0,07} \\
\hline$\Delta$ & 7 & & 2,03 & & $45 \mid$ & & & & & \\
\hline \multirow[t]{2}{*}{ Vmin - Vmaks } & 28 - & 35 & 8,12 & \begin{tabular}{|l|l|}
- & 10,15 \\
\end{tabular} & \begin{tabular}{l|l|l|}
$115 \cdot$ & 140 \\
\end{tabular} & 50,75 & \multirow{2}{*}{18,84} & \multirow{2}{*}{38,81} & \multirow{2}{*}{3,26} & \multirow{2}{*}{0,07} \\
\hline & 7 & & 2,03 & & 25 & & & & & \\
\hline \multirow[t]{2}{*}{ Vmaks-Vstate } & 35 . & 35 & 10,15 & \begin{tabular}{|l|l|} 
& 10,15 \\
\end{tabular} & $140 \cdot-180$ & \multirow{3}{*}{406,00} & \multirow{3}{*}{18,84} & \multirow{3}{*}{970,34} & \multirow{3}{*}{81,61} & \multirow{3}{*}{1,70} \\
\hline & 0 & & 0,00 & & $40 \mid$ & & & & & \\
\hline Total Vstate & 35 & & & 10,15 & 40 & & & & & \\
\hline
\end{tabular}

Table 6. Data of energy requirement Vmax $\omega 35$ dan Vmin $\omega 26$

\begin{tabular}{|c|c|c|c|c|c|c|c|c|c|c|c|}
\hline \multirow{2}{*}{ Kondisi Be rkendara } & \multicolumn{2}{|c|}{$v_{\omega}$} & \multicolumn{2}{|c|}{ Vlinear } & \multicolumn{2}{|c|}{ Waktu (t) } & $\mathrm{s}$ & Inersia (I) & \multicolumn{2}{|c|}{$\mid$ Kinetik (f) $\mid$ Daya (P) } & Arus (I) \\
\hline & \multicolumn{2}{|c|}{ (radsec) } & \multicolumn{2}{|c|}{ (m/s) } & \multicolumn{2}{|c|}{ (s) } & $1 \mathbf{x}(\mathrm{m})$ & $\mathrm{Kgm}^{\wedge} \mathbf{2}$ & Joule & Watt & A \\
\hline \multirow[t]{2}{*}{0 -Vmaks } & 0 & 35 & 0 & \begin{tabular}{|l|l|}
- & 10,15 \\
\end{tabular} & 0 & 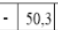 & 510,85 & \multirow{2}{*}{18,84} & \multirow{2}{*}{970,34} & \multirow{2}{*}{81,61} & \multirow{2}{*}{1,70} \\
\hline & 35 & & 10,15 & & 50,3 & & & & & & \\
\hline \multirow[t]{2}{*}{ Vmaks - Vstate } & 35 & 35 & 10,15 & \begin{tabular}{|l|l|}
- & 10,15 \\
\end{tabular} & 50,3 & $\begin{array}{l}-70 \\
\end{array}$ & \multirow{3}{*}{199,65} & \multirow{3}{*}{18,84} & \multirow{3}{*}{970,34} & \multirow{3}{*}{81,61} & \multirow{3}{*}{1,70} \\
\hline & 0 & & 0,00 & & 19,7 & & & & & & \\
\hline Total Vstate & \multicolumn{2}{|c|}{35} & \multicolumn{2}{|c|}{10,15} & \multicolumn{2}{|c|}{19.67} & & & & & \\
\hline \multirow[t]{2}{*}{ Vstate - Vmin } & 35 & 26 & 10,15 & 7,54 & 70 & \begin{tabular}{|l|l|}
- & 123 \\
\end{tabular} & 138,33 & \multirow{2}{*}{18,84} & \multirow{2}{*}{64,16} & \multirow{2}{*}{5,40} & \multirow{2}{*}{0,11} \\
\hline & 9 & & 2,61 & & 53 & & & & & & \\
\hline \multirow[t]{2}{*}{ Vmin - Vmaks } & 26 & 35 & 7,54 & \begin{tabular}{|l|l|}
- & 10,15 \\
\end{tabular} & 123 & \begin{tabular}{l|l|}
- & 151 \\
\end{tabular} & 73,08 & \multirow{2}{*}{18,84} & \multirow{2}{*}{64,16} & \multirow{2}{*}{5,40} & \multirow{2}{*}{0,11} \\
\hline & 9 & & 2,61 & & 28 & & & & & & \\
\hline \multirow[t]{2}{*}{ Vmaks - Vstate } & 35 & 35 & 10,15 & \begin{tabular}{|l|l|}
- & 10,15 \\
\end{tabular} & 151 & 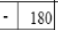 & \multirow{3}{*}{294,35} & \multirow{3}{*}{18,84} & & & \\
\hline & 0 & & 0,00 & & 29 & & & & 970,34 & 81,61 & 1,70 \\
\hline Total Vstate & 3. & & & 10,15 & & 29 & & & & & \\
\hline
\end{tabular}

Table 7. Summary of Simulation Result

\begin{tabular}{|c|c|c|c|c|c|c|}
\hline \\
\hline V.Maks (m/s) & V.Min $(m / s)$ & $\begin{array}{c}\text { V.Average } \\
(\mathrm{m} / \mathrm{s})\end{array}$ & $\begin{array}{c}\text { Total Daya } \\
\text { (Watt) }\end{array}$ & Arus (A) & $\begin{array}{l}\text { Total Jarak } \\
\text { (m) }\end{array}$ & $\begin{array}{l}\text { Total Waktu } \\
\text { (s) }\end{array}$ \\
\hline \multirow[t]{3}{*}{$11,02 \mathrm{~m} / \mathrm{s}$} & $6,96 \mathrm{~m} / \mathrm{s}$ & $8,99 \mathrm{~m} / \mathrm{s}$ & $314,70 \mathrm{~W}$ & $6,56 \mathrm{~A}$ & $1265 \mathrm{~m}$ & $180 \mathrm{~s}$ \\
\hline & $6,67 \mathrm{~m} / \mathrm{s}$ & $8,85 \mathrm{~m} / \mathrm{s}$ & $318,56 \mathrm{~W}$ & $6,64 \mathrm{~A}$ & $1275 \mathrm{~m}$ & $180 \mathrm{~s}$ \\
\hline & $5,8 \mathrm{~m} / \mathrm{s}$ & $8,41 \mathrm{~m} / \mathrm{s}$ & $331,75 \mathrm{~W}$ & $6,91 \mathrm{~A}$ & $1344 \mathrm{~m}$ & $180 \mathrm{~s}$ \\
\hline V.Maks (m/s) & V.Min $(m / s)$ & $\begin{array}{c}\text { V.Average } \\
(\mathrm{m} / \mathrm{s})\end{array}$ & $\begin{array}{l}\text { Total Daya } \\
\text { (Watt) }\end{array}$ & Arus (A) & $\begin{array}{l}\text { Total Jarak } \\
\text { (m) }\end{array}$ & $\begin{array}{l}\text { Total Waktu } \\
\text { (s) }\end{array}$ \\
\hline \multirow[t]{3}{*}{$10,15 \mathrm{~m} / \mathrm{s}$} & $8,7 \mathrm{~m} / \mathrm{s}$ & $9,42 \mathrm{~m} / \mathrm{s}$ & $248,15 \mathrm{~W}$ & 5,17 & $1357 \mathrm{~m}$ & $180 \mathrm{~s}$ \\
\hline & $8,12 \mathrm{~m} / \mathrm{s}$ & $9,13 \mathrm{~m} / \mathrm{s}$ & $251,35 \mathrm{~W}$ & 5,24 & $1259 \mathrm{~m}$ & $180 \mathrm{~s}$ \\
\hline & $7,54 \mathrm{~m} / \mathrm{s}$ & $8,84 \mathrm{~m} / \mathrm{s}$ & $255,61 \mathrm{~W}$ & 5,33 & $1216 \mathrm{~m}$ & $180 \mathrm{~s}$ \\
\hline
\end{tabular}

\section{CONCLUSION}

Conclusions can be drawn as follows :

1. The electric energy needs of GARNESA cars with an average speed selection of $9.42 \mathrm{~m} / \mathrm{s}$ based on a maximum speed of $10.15 \mathrm{~m} / \mathrm{s}$ and a minimum speed of $8.70 \mathrm{~m} / \mathrm{s}$ are $248.15 \mathrm{Watts}$ (travel time of 180 seconds / lap). Distance of $1357 \mathrm{~m}$.

2. The driver's driving strategy based on the average speed of $9.42 \mathrm{~m} / \mathrm{s}$ consumes more energy savings and the distance traveled further. 


\section{REFERENCES}

[1] Daziano R. Conditional-logit Bayes estimators for consumer valuation of electric vehicle driving range. Resource Energy Econ 2013; 35(3): 429-450.

[2] Pamungkas (2015). Dynamics Modeling and Simulation of Generation II Electric Car Handling Handling. Proceedings of the XIV International Mechanical Engineering Seminar.

[3] Rauh, N., Franke, T., Krems, J. F., \& Universität, T. (2012). Understanding the Impact of Electric Vehicle Driving Experience on Range Anxiety. https://doi.org/10.1177/0018720814546372

[4] Tadjudin Muhammad (2017). Driving Strategy for Electric Cars at Energy Efficient Car Contests. Proceedings of the XVI Annual Mechanical Engineering Seminar (SNTTM XVI) 5-6 October 2017.

[5] Walsh C, Carroll A, Eastlakem A and Blythe P. Electric vehicle driving style and duty variation performance study. Report, Cenex, Loughborough, Leicestershire,UK, 2010.

[6] Y. Hori, Future vehicle driven by electricity and control (Research on four wheel motored UOT Electric March II), Proceedings of the 7th International Workshop on Advanced Motion Control, Maribor, Slovenia, 2002, pp. 1-4

[7] Z. Cai, C. Ma, and Q. Zhao, Acceleration to torque ratio based anti-kid control for electric vehicles, International Conference on Mechatronics and Embedded System and Appalications, Wuhan, China, 2010, pp. 577-581. 